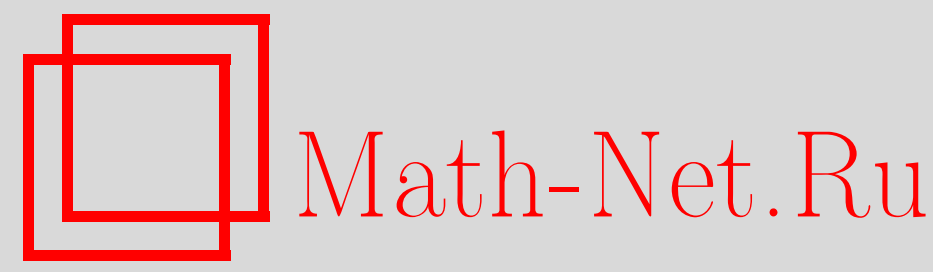

Н. Н. Ганиходжаев, У. А. Розиков, Групповое представление леса Кэли и его некоторые применения, Изв. РАН. Сер. матем., 2003, том 67, выпуск 1, 21-32

DOI: https://doi.org/10.4213/im416

Использование Общероссийского математического портала Math-Net.Ru подразумевает, что вы прочитали и согласны с пользовательским соглашением

http://www.mathnet.ru/rus/agreement

Параметры загрузки:

IP: 52.90 .164 .192

26 апреля 2023 г., 08:42:10 
УДК $517.98+530.1$

\author{
Н. Н. Ганиходжаев, У. А. Розиков
}

\title{
Групповое представление леса Кэли и его некоторые применения
}

\begin{abstract}
Лес и произведения деревьев Кэли порядка $k \geqslant 1$ представлены как подгруппы группы свободного произведения $m(m>k)$ циклических групп второго порядка. Определены группы автоморфизмов этих объектов. Для модели Изинга на лесе Кэли даны полные описания множеств трансляционно-инвариантных и периодических гиббсовских мер. Построен новый класс предельных гиббсовских мер неоднородной модели Изинга на дереве Кэли. Найдены достаточные условия невозвратности случайного блуждания в периодической случайной среде на лесе, когда размеры скачков блуждающей частицы ограничены.

Библиография: 13 наименований.
\end{abstract}

1. Введение. В настоящее время теория графов стала простым, доступным и мощным средством решения многих задач. В некоторых ситуациях полезно рассматривать помеченные графы, т.е. графы, вершинам которых присвоены некоторые метки, например номера $1,2,3, \ldots$.

Если граф бесконечен, то помечание графа становится нелегкой задачей.

В некоторых задачах целесообразно то, что метки образуют группу. Например, для описания предельных гиббсовских мер, определенных на графах, необходимо определить групповое представление и автоморфизмы графа (см. [1]-[6]).

В настоящей работе определяются и изучаются некоторые группы преобразований бесконечного леса и произведений бесконечных деревьев Кэли, включая группы автоморфизмов этих объектов.

Под деревом Кәли $\Gamma^{k}$ порядка $k \geqslant 1$ понимается связный граф без циклов, в котором степень каждой вершины равна $k+1$.

Любой граф̆ без циклов называется лесом.

Если лес содержит счетное число компонентов, каждый из которых является деревом Кэли порядка $k \geqslant 1$, то его назовем лесом Кәли и обозначим $\Lambda_{k}$.

Если из каждой вершины дерева Кэли порядка $k \geqslant 1$ вырастет дерево Кэли порядка $k$, то полученный граф (дерево) назовем произведением деревьев Кэли и обозначим через $\Gamma^{k} \odot \Gamma^{k}$ (см. рис. 1$)$.

ПРЕДЛОЖЕНИЕ 1 [1]. Существует взаимно однозначное соответствие между множеством вериин $V$ дерева Кәли порядка $k$ и группой $G_{k}$ свободного произведения $k+1$ чиклических групп второго порядка с образующими $a_{1}, a_{2}, \ldots, a_{k+1}$ соответственно.

Для полноты изложения кратко приведем описание этого соответствия: произвольной фиксированной вершине $x_{0} \in V$ поставим в соответствие единичньй

(C) Н.Н. ГАниходЖАев, У.А. РОзиков, 2003 


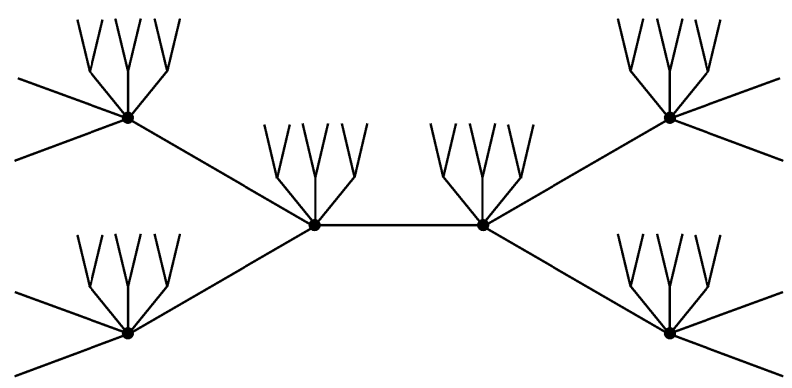

Рис. 1

элемент $е$ группы $G_{k}$. Так как рассматриваемый граф̆ без ограничения обшности можно считать плоским, занумеруем соседние с $x_{0}$ вершины, двигаясь в положительном направлении (см. рис. 2).

Каждой вершине $x_{i} \in V$ поставим в соответствие образующую $a_{i}, i=1,2, \ldots$ $\ldots, k+1$. Теперь для каждой вершины $x_{i} \in V$ определим двойную нумерацию $x_{i j}$ соседних вершин $x_{i}$. Так как одна из соседних вершин вершины $x_{i}$ есть $x_{0}$, то положим $x_{i i}=x_{0}$, и тогда нумерация остальных соседних вершин $x_{i} \in V$ производится однозначно по приведенному ранее правилу нумерации. Каждой вершине $x_{i j}$ поставим в соответствие слово $a_{i} a_{j}, i, j=1,2, \ldots, k+1$. Так как $x_{i i}=x_{0}$ и $a_{i}^{2}=e$, то это соответствие согласуется с предущим шагом. Далее, для соседних вершин $x_{i j}$ определим тройную нумерацию следуюшим образом. Так как одна из соседних для $x_{i j}$ вершин есть $x_{i}$, то положим $x_{i j j}=x_{i}$, и тогда нумерация остальных соседних вершин производится однозначно. Каждой вершине $x_{i j k}$ поставим в соответствие слово $a_{i} a_{j} a_{k}$. Это соответствие согласуется с предыдушимм шагом, так как $x_{i j j}=x_{i}$ и $a_{i} a_{j} a_{j}=a_{i} a_{j}^{2}=a_{i}$.

Таким образом, можно установить взаимно однозначное соответствие между множеством вершин дерева Кэли $\Gamma^{k}$ и группой $G_{k}$. Предложение доказано.

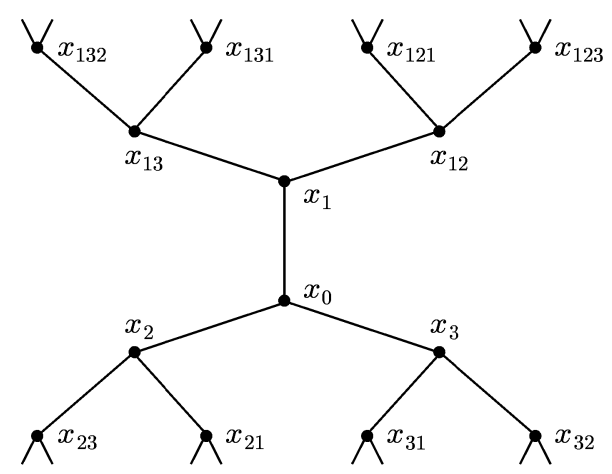

Рис. 2

Представление, построенное выше, называется $п р а в ы . м$ так как в этом случае, если $x$ и $y$-соседние вершины, a $g, h \in G_{k}$ - соответствуюшие им элементы групшы, то либо $g=h a_{i}$, либо $h=g a_{j}$ для некоторых $i$ и $j$. Аналогично определяется левое представление. 
Ниже мы покажем, что множеству вершин леса $\Lambda_{k}$ и леса $F^{k}$ (где $F^{k}$ состоит из счетного числа связанных компонент $\Gamma^{k} \odot \Gamma^{k}$ и $\Gamma^{k}$ ) можно естественным образом поставить в соответствие некоторую подгруппу $G$ группы $G_{k}$, что позволит явно определить группы преобразований $\Lambda_{k}$ и $F^{k}$.

В работе также рассматриваются следующие задачи:

1) описание периодических гиббсовских мер на $\Lambda_{k}$;

2) описание новых (не совпадающих с известными) гиббсовских мер для неоднородной модели Изинга на дереве Кэли с использованием результатов п. 1);

3 ) определение и изучения случайных блужданий в случайной среде на $F^{k}$.

2. Описание некоторых свойств подгрупп группы $G_{k}$. Пусть $G$ - произвольная подгруппа групшы $G_{k}$.

Обозначим $I(G)=G \cap\left\{a_{1}, a_{2}, \ldots, a_{k+1}\right\}$. Пусть $G(I)$ - свободное произведение циклических групп $A_{i}=\left\{e, a_{i}\right\}$, где $e$ - единичный элемент и $a_{i} \in I(G)$.

Определим на $G$ бинарное отношение $\sim$, положив $x \sim y$ для $x, y \in G$ тогда и только тогда, когда в $G(I)$ существует такое слово $z$, что $x=y z$. Очевидно, что отношение $\sim$ является отношением эквивалентности и, следовательно, множество $G$ разбивается на классы смежности $G_{x}=\{y \in G: y=x z, z \in G(I)\}$.

ЛЕмма 1. Для любого $x \in G$ справедливы следующие имликации:

1) $I(G)=\varnothing \Longleftrightarrow G_{x}=\{x\}$;

2) $I(G)=\left\{a_{i}\right\} \Longleftrightarrow G_{x}=\left\{x, x a_{i}\right\}, \quad i=1,2, \ldots, k+1$;

3) $|I(G)|>1 \Longleftrightarrow\left|G_{x}\right|=\infty$, где $|\cdot|-$ число әлементов множества.

ДокаЗАтЕльство. Заметим, что если $x \in G$, то для того, чтобы $x a_{i} \in G$, необходимо и достаточно, чтобы $a_{i} \in G$. Откуда следует доказательство леммы.

Пусть отображение $\nu: G_{k} \rightarrow\left\{a_{1}, a_{2}, \ldots, a_{k+1}\right\}$ определено по формуле $\nu(x)=$ $\nu\left(a_{i_{1}} a_{i_{2}} \ldots a_{i_{n}}\right)=a_{i_{n}}$ и $\nu(e) \notin I(G)$ - произвольное.

Теорема 1. Для любого $x \in G$ существует единственное $u(x) \in G_{x}$ такое, что $и(x) \nu(u(x)) \notin G$.

ДокаЗАТЕЛЬСТво. Существование. Пусть $y \in G_{x}$; тогда $y=x a_{i_{1}} a_{i_{2}} \ldots a_{i_{n}}$, где $a_{i_{j}} \in I(G), j=1,2, \ldots, n$. С другой стороны, элемент $x$ можно представить как $x=x^{*} a_{j_{1}} a_{j_{2}} \ldots a_{j_{m}}$, где $a_{j_{s}} \in I(G), s=1,2, \ldots, m$, и $x^{*}$ такое, что $x^{*} \in$ $G, \nu\left(x^{*}\right) \notin G$. Действительно, $x^{*}$ определяется следуюшим образом: если $x$ удовлетворяет условию $\nu(x) \notin G$, то $x^{*}=x$; в случае $\nu(x) \in G$ рассмотрим $x_{1}=x \nu(x)$ и проверим условие $\nu\left(x_{1}\right) \notin G$; если оно вьполняется, то в качестве $x^{*}$ берем $x_{1}$, в противном случае рассмотрим $x_{2}=x_{1} \nu\left(x_{1}\right)$ и проверим условие $\nu\left(x_{2}\right) \notin G$, и т.д. Этот процесс продолжается до тех пор, пока не выполняется нужное нам условие. Так как $\nu(e) \notin I(G)$, то этот процесс заканчивается, по крайней мере, в случае $x^{*}=e$ (в случае $x \sim e$ ). Если $x$ не эквивалентно $e$, то для определения $x^{*}$ будет сделано не более $|x|$ шагов.

Из $\nu\left(x^{*}\right) \notin G$ следует, что $x^{*} \nu\left(x^{*}\right) \notin G$. Таким образом, $u(x)=x^{*}$.

Eдинственность. Пусть существуют $u_{1}(x), u_{2}(x) \in G_{x}$. Тогда для любого $y$ имеем

$$
y=u_{1}(x) a_{i_{1}} a_{i_{2}} \ldots a_{i_{n}}, \quad y=u_{2}(x) a_{j_{1}} a_{j_{2}} \ldots a_{j_{m}}
$$


откуда $u_{1}(x)=u_{2}(x) a_{j_{1}} \ldots a_{i_{1}}$. Если $a_{j_{1}} \ldots a_{i_{1}}=e$, то это противоречит тому, что $u_{1}(x) \neq u_{2}(x)$, если $a_{j_{1}} \ldots a_{i_{1}} \neq e$, то это противоречит тому, что $\nu\left(u_{1}(x)\right) \notin G$. Теорема доказана.

ОПРЕДЕЛЕНИЕ 1. Элемент $u(x) \in G_{x}$, определяемый в теореме 1 , назовем $к о p$ нем класса $G_{x}$.

В дальнейшем запись $G_{x}$ означает, что $x$ - корень класса $G_{x}$.

Теорема 2. Любая подгруппа $G \subset G_{k}$ имеет счетное число корневых әлементов, если $G \neq G(I)$.

ДокАЗАТЕЛЬСТво. В силу теоремы 1 существует хотя бы одно корневое слово $u$. Заметим, что $y=x u$ также является корневым, где $x \in G$. Так как $G-$ счетное множество, то отсюда следует доказательство теоремы.

СлЕДСТВИЕ 1. Любая подгруппа $G(G \neq G(I))$ разбивается на счетное иисло классов эквивалентных әлементов.

Обозначим через $K(G)$ множество всех корней $G$. Очевидна следующая лемма.

Лемма 2. Пусть $u \in K(G)$. Тогда:

1) $u a_{i} \notin K(G)$ для любого $i=1,2, \ldots, k+1$;

2) если $е \neq u \in K(G)$, то для того, чтобь $a_{i} u \in K(G)$, необходимо $и$ достаточно, чтобы $a_{i} \in G$.

Определим на $G \subset G_{k}$ структуру графа следуюшим образом: вершины, соответствуюшие словам $x, y \in G$, назовем ближсайшими соседями и соединим ребром, если либо $x=y a_{i}$, либо $y=x a_{j}$ для некоторого $i$ или $j$.

Нетрудно убедиться в том, что определенный таким образом граф̆ образует лес деревьев Кэли порядка $|I(G)|-1$, т.е. $\Lambda_{|I(G)|-1}$.

3. Групповое представление леса Кэли. Пусть $\Lambda_{k}-$ лес Кэли и $G$ - подгрупша групшы $G_{m}$, где $m>k$.

ПРЕДЛОЖЕНИЕ 2. Если $|I(G)|=k+1$, то существует взаимно однозначное соответствие между мноэсеством вершин $\Lambda$ леса Кәли $\Lambda_{k}$ и подгрупnой $G$.

ДокаЗАТЕЛЬСтво. Так как $\Lambda_{k}$ содержит счетное число компонентов и $G-$ счетное число классов эквивалентных элементов, то существует естественное взаимно однозначное соответствие между классами $G_{x}, x \in K(G)$, и компонентами $\Lambda_{k}^{(i)}, i=1,2, \ldots$, леса $\Lambda_{k}$. В силу предложения 1 сушествует однозначное соответствие между множеством вершин $\Lambda_{k}^{(i)}$ и элементами класса $G_{x}$. Предложение доказано.

Представление, построенное вьше, назовем правым, так как в этом случае если $x, y$ - соседние вершины, а $g, h \in G$ - соответствуюшие им элементы группы, то либо $g=h a_{i}$, либо $h=g a_{j}$ для некоторого $i$ или $j$. Аналогично определяется левое представление. 
4. Групповое представление леса $F^{k}$. Пусть $G \subset G_{m}$ - подгруппа такая, что $|I(G)|=k+1$, где $m>k$. Определим на $K(G) \backslash\{e\}$ структуру графа следуюшим образом: вершины, соответствуюшие словам $u, v \in K(G) \backslash\{e\}$, назовем ближайшими соседями и соединим ребром, если либо $u=a_{i} v$, либо $v=a_{j} u$ для некоторого $i$ или $j$. В силу леммы 2 заметим, что определенный таким образом граф̆ образует лес Кэли порядка $|I(G)|-1$, но с левым представлением $K(G) \backslash\{e\}$, которое, вообще говоря, не является группой.

Структуру графа на $G$ определим, как в п. 2, и каждому корню добавим ребра, которые построены на $K(G) \backslash\{e\}$. Тогда полученный граф является $F^{k}$.

Таким образом, $G$ c ранее определенной на нем структурой графа является групповым представлением $F^{k}$.

5. Определения некоторых автоморфизмов подгруппы $G$ группы $G_{k}$. Рассмотрим на группе $G$ следующие преобразования.

1) Преобразования левого (правого) сдвигов, определяемые следующим образом: для $g_{0} \in G$ положим

$$
T_{g_{0}}(h)=g_{0} h \quad\left(T_{g_{0}}^{*}(h)=h g_{0}\right) \quad \text { для любого } h \in G .
$$

Совокупность всех левых (правых) сдвигов на $G$ изоморфна группе $G$.

2) Преобразование $G$, определенное следуюшим образом: для $g_{0} \in G$

$$
T_{g_{0}}(h)=g_{0}^{-1} h g_{0} \quad \text { для любого } h \in G,
$$

называется внутренним автоморфизмом группь $G$.

Ясно, что любое преобразование $S$ группы $G$ индуцирует преобразование $S^{*}$ на множестве вершин $\Lambda_{k}$ и $F^{k}$.

6. Полное описание периодических гиббсовских мер модели Изинга на $\Lambda_{k}$. Пусть $G$-групповое представление леса $\Lambda_{k}$ и $A \subset G$-конечное подмножество. Обозначим через $\vee_{A}=\{-1,1\}^{A}$ пространство конфигураций на множестве $A$, т.е. множество всевозможных функций, определенных на $A$ и принимаюших два значения: $-1,1$.

В модели Изинга на $\Lambda_{k}$ гамильтониан $H: \vee_{A} \rightarrow \mathbb{R}$ имеет вид

$$
H(\sigma)=-J \sum_{\langle x, y\rangle} \sigma(x) \sigma(y),
$$

где суммирование ведется по всем парам ближайших соседей $\langle x, y\rangle$ и $J \in \mathbb{R}$.

Для каждого слова $x \in G$ обозначим через $|x|$ его длину, т.е. число букв в несократимой записи $x$. Введем обозначения:

$$
W_{n}=\{x \in G:|x|=n\}, \quad V_{n}=\{x \in G:|x| \leqslant n\}, \quad L_{n}=\left\{\langle x, y\rangle: x, y \in V_{n}\right\} .
$$

Пусть $h_{x}$ - действительнозначная функция, $x \in G$. Для каждого $n>0$ рассмотрим меру на $\vee_{V_{n}}$, определенную формулой

$$
\mu_{n}\left(\sigma_{n}\right)=Z_{n}^{-1} \exp \left\{J \beta \sum_{\langle x, y\rangle \in L_{n}} \sigma(x) \sigma(y)+\sum_{x \in W_{n}} h_{x} \sigma(x)\right\},
$$


где $\beta=\frac{1}{T}$ ( $T>0$ - температура), $\sigma_{n}=\left\{\sigma(x): x \in V_{n}\right\} \in \vee_{V_{n}}$ и $Z_{n}$ - нормирующий делитель.

Условия согласования для $\mu_{n}\left(\sigma_{n}\right), n>0$, определяются равенством

$$
\sum_{\sigma^{(n)}} \mu_{n}\left(\sigma_{n-1}, \sigma^{(n)}\right)=\mu_{n-1}\left(\sigma_{n-1}\right),
$$

где $\sigma^{(n)}=\left\{\sigma(x): x \in W_{n}\right\}$.

Обозначим $S(x)=\left\{y \in W_{|x|+1}:\langle x, y\rangle\right\}$.

ТЕОРЕМА 3. Для того чтобы меры (1) удовлетворяли условию (2), необходимо и достаточно, чтобь для $h_{x}, x \in G$, выполнялось равенство

$$
h_{x}=\sum_{y \in S(x)} f\left(h_{y} ; \theta\right)
$$

əде $-1<\theta=\operatorname{th}(h \beta)<1, \quad f(x ; \theta)=\operatorname{arcth}(\theta \operatorname{th} x)$.

ДокАЗАТЕЛьСтво. Подставляя формулу (1) в (2), заметим, что равенства (2) и (3) эквивалентны.

Очевидны следующие свойства функции $f$ :

(a) $f(-x ; \theta)=-f(x ; \theta)$;

(b) $\lim _{x \rightarrow \infty} f(x ; \theta)=\operatorname{arcth} \theta$;

(c) $f_{x}^{\prime}(0 ; \theta)=\theta, f_{x}^{\prime}(x ; \theta) \leqslant|\theta|$;

(d) $f_{x x}^{\prime \prime}(x ; \theta)<0$, если $x>0, \theta>0$.

Таким образом, в силу теоремы Колмогорова из теоремы 3 следует, что каждой совокупности величин $\left\{h_{x} ; x \in G\right\}$, удовлетворяющей $(3)$, соответствует единственная предельная мера $\mu$ на $\vee_{G}$ такая, что $\mu\left(\sigma_{n}\right)=\mu_{n}\left(\sigma_{n}\right)$.

ОПРЕДЕЛЕНИЕ 2. Совокупность чисел $\left\{h_{x} ; x \in G\right\}$ назовем $H_{0}$-nериодической (где $H_{0}$ - нормальный делитель группы $G$ ), если $h_{x}=h_{y x}$ для любых $x \in G$ и $y \in H_{0}$.

ОПРЕДЕЛЕНИЕ 3 . Меру, соответствующую $H_{0}$-периодической совокупности величин, назовем $H_{0}$-периодической. G-периодическая мера называется трансляционно-инвариантной.

Пусть $H_{0}$ - нормальный делитель индекса $r$ группы $G$ и $G / H_{0}=\left\{H_{0}, H_{1}, \ldots\right.$ $\left.\ldots, H_{r-1}\right\}$ - факторгруппа. Введем обозначения:

$$
q_{i}(x)=\left|S(x) \cap H_{i}\right|, \quad i=0,1, \ldots, r-1 ; \quad N(x)=\left|\left\{j: q_{j}(x) \neq 0\right\}\right|,
$$

где $|\cdot|$ - число элементов множества,

$$
Q(x)=\left(q_{0}(x), q_{1}(x), \ldots, q_{r-1}(x)\right) .
$$

Заметим (см. [5]), что для любого $x \in G$ сушествует перестановка $\pi_{x}$ координат вектора $Q(x)$ такая, что

$$
\pi_{x} Q(e)=Q(x) .
$$


Из равенства (4) следует, что $N(x)=N(e)$ для любого $x \in G$.

$H_{0}$-периодическая совокупность величин имеет следующий вид:

$$
\left\{h_{x}=h_{i}, \text { если } x \in H_{i}, \quad i=0,1, \ldots, r-1\right\} \text {. }
$$

В силу теоремы 3 и равенства (4) величины $h_{m}$ удовлетворяют системе уравнений

$$
h_{m}=\sum_{j=1}^{N(e)} q_{i_{j}}(e) f\left(h_{\pi_{m}\left(i_{j}\right)} ; \theta\right)-f\left(h_{\pi_{m}\left(i_{j_{0}}\right)} ; \theta\right) \text {, }
$$

где $m=\overline{0, r-1}, j_{0}=\overline{1, N(e)}, N(e)=\left|\left\{i_{1}, i_{2}, \ldots, i_{N(e)}\right\}\right|$. Пусть $\widetilde{G}-$ подгруппа групшы $G$, состояшая из слов четной длины. Очевидно, что $\widetilde{G}$ является подгруппой индекса два.

ТЕОРема 4. Для любого нормального делителя конечного индекса $H_{0}$ всякая $H_{0}$-периодическая мера Гиббса совпадает либо с транслячионно-инвариантной, либо с $\widetilde{G}$-периодической мерой.

ДОКАЗАТЕЛЬСТВО. Из уравнений (5) получим

$$
f\left(h_{\pi_{m}\left(i_{1}\right)} ; \theta\right)=f\left(h_{\pi_{m}\left(i_{2}\right)} ; \theta\right)=\cdots=f\left(h_{\pi_{m}\left(i_{N(e)}\right)} ; \theta\right)
$$

Отсюда имеем

$$
h_{\pi_{m}\left(i_{1}\right)}=h_{\pi_{m}\left(i_{2}\right)}=\cdots=h_{\pi_{m}\left(i_{N(e)}\right)} .
$$

Следовательно,

$$
\begin{aligned}
& h_{x}=h_{y}=h_{1}, \text { если } x, y \in \widetilde{S}(z), \quad z \in \widetilde{G}, \\
& h_{x}=h_{y}=h_{2}, \text { если } x, y \in \widetilde{S}(z), \quad z \in G \backslash \widetilde{G},
\end{aligned}
$$

где $\widetilde{S}(z)$ - множество ближайших соседей точки $z \in G$. Следовательно, если $h_{1}=h_{2}$, то меры будут трансляционно-инвариантными, если $h_{1} \neq h_{2}$, то меры являются $\widetilde{G}$-периодическими. Теорема доказана.

Пусть $H_{0}$ - нормальный делитель конечного индекса группы $G$. Возникает вопрос: при каком условии на $H_{0}$ всякая $H_{0}$-периодическая мера Гиббса является транслящионно-инвариантной? Пусть $I\left(H_{0}\right)=H_{0} \cap\left\{a_{1}, a_{2}, \ldots, a_{k+1}\right\}$.

ТЕОРема 5. Если $I\left(H_{0}\right) \neq \varnothing$, то всякая $H_{0}$-периодическая мера Гиббса для модели Изинга является трансляционно-инвариантной. 
ДоКАЗАТЕЛЬСТВо. Заметим, что если $x \in H_{0}$, то для того, чтобы $x a_{i} \in H_{0}$, необходимо и достаточно, чтобы $a_{i} \in H_{0}$. Так как $I\left(H_{0}\right) \neq \varnothing$, то сушествует $a_{i} \in$ $H_{0}$. Следовательно, $H_{0}$ содержит подмножество $H_{0} a_{i}=\left\{x a_{i}: x \in H_{0}\right\}$. В силу теоремы 4 имеем $h_{x}=h_{1}$ и $h_{x a_{i}}=h_{2}$, но так как $x$ и $x a_{i}$ принадлежат классу $H_{0}$, получим $h_{x}=h_{x a_{i}}=h_{1}=h_{2}$. Таким образом, всякая $H_{0}$-периодическая мера Гиббса является транслящионно-инвариантной. Теорема доказана.

В силу теорем 4,5 задача описания $H_{0}$-периодических гиббсовских мер при $I\left(H_{0}\right) \neq \varnothing$ сводится к описанию неподвижных точек отображения

$$
h=\left(\left|I\left(H_{0}\right)\right|-1\right) f(h ; \theta)
$$

и при $I\left(H_{0}\right)=\varnothing$ сводится к описанию решения системы

$$
\begin{aligned}
& h_{1}=\left(\left|I\left(H_{0}\right)\right|-1\right) f\left(h_{2} ; \theta\right), \\
& h_{2}=\left(\left|I\left(H_{0}\right)\right|-1\right) f\left(h_{1} ; \theta\right) .
\end{aligned}
$$

Из свойств (a)-(d) функции $f$ следует, что уравнение (6) имеет единственное решение $h=0$, если $|\theta|<\frac{1}{\left|I\left(H_{0}\right)\right|-1}$, и три решения $h=0, \pm h_{*}\left(h_{*}>0\right)$, если $1>|\theta| \geqslant \frac{1}{\left|I\left(H_{0}\right)\right|-1}$.

Система (7) имеет единственное решение $(0,0)$, если $|\theta|<\frac{1}{\left|I\left(H_{0}\right)\right|-1}$, три решения $(0,0),\left(-h_{*},-h_{*}\right),\left(h_{*}, h_{*}\right)$, если $1>\theta \geqslant \frac{1}{\left|I\left(H_{0}\right)\right|-1}$, и три решения $(0,0)$, $\left(-h_{*}, h_{*}\right),\left(h_{*},-h_{*}\right)$, если $-1<\theta \leqslant-\frac{1}{\left|I\left(H_{0}\right)\right|-1}$.

Обозначим через $\mu^{+}, \mu^{-}, \mu^{0}$ (или $\mu^{ \pm}, \mu^{\mp}, \mu^{0}$ ) меры Гиббса, соответствуюшие совокупностям $\left\{h_{x}=h_{*}, x \in G\right\},\left\{h_{x}=-h_{*}, x \in G\right\},\left\{h_{x}=0, x \in G\right\}$ (или

$$
\begin{aligned}
& h_{x}=\left\{\begin{array}{lll}
h_{*}, & \text { если } & x \in \widetilde{G}, \\
-h_{*}, & \text { если } & x \in G \backslash \widetilde{G},
\end{array}\right. \\
& h_{x}=\left\{\begin{array}{lll}
-h_{*}, & \text { если } & x \in \widetilde{G}, \\
h_{*}, & \text { если } & x \in G \backslash \widetilde{G},
\end{array}\right.
\end{aligned}
$$

$\left.\left\{h_{x}=0, x \in G\right\}\right)$.

Таким образом, применяя теоремы 4,5 и учитывая анализ решений уравнений (6), (7), мы доказали следующую теорему.

Теорема 6. Для модели Изинга на лесе Кәли относительно любого нормального делителя конечного индекса $H_{0}$ при $1>\theta \geqslant \frac{1}{\left|I\left(H_{0}\right)\right|-1} \quad(-1<\theta \leqslant$ $\left.-\frac{1}{\left|I\left(H_{0}\right)\right|-1}\right)$ существуют только три периодические мерь Гиббса $\mu^{+}, \mu^{-}$, $\mu^{0}\left(\mu^{ \pm}, \mu^{\mp}, \mu^{0}\right)$. Более того, меры $\mu^{+}, \mu^{-} \mu^{0}$ являются трансляционно-инвариантными и меры $\mu^{ \pm}, \mu^{\mp}$ являются $\widetilde{G}$-периодическими.

ЗАмЕчАниЕ 1. Полное описание периодических гиббсовских мер означает описание таких мер относительно любого нормального делителя конечного индекса.

ЗАмечаниЕ 2. Используя периодические меры, полученные в теореме 6 , можно построить несчетное число непериодических гиббсовских мер. Аналогичные реультаты для некоторых моделей на дереве Кэли получены в работах [3], [5]-[7], а наш результат получен для модели Изинга на лесе Кэли. 
ЗАмЕчАниЕ 3. Если мы выберем на каждой компоненте леса Кэли некоторые периодические меры (которые сушествуют на дереве Кэли, см. [1], [3], [5]-[9]) и если хотя бы две компоненты этих мер различны, то полученная комбинационная мера на лесе уже не будет периодической, так как при сдвиге по нормальньм делителям с одной компоненты на другую значения функции $h_{x}$ не совпадают.

7. Новые меры Гиббса неоднородной модели Изинга на дереве Кэли. Неоднородная модель Изинга на дереве Кэли задается гамильтонианом

$$
H(\sigma)=-\sum_{\langle x, y\rangle} J_{\langle x, y\rangle} \sigma(x) \sigma(y),
$$

где суммирование ведется по всем парам ближайших соседей $\langle x, y\rangle$ и $J_{\langle x, y\rangle} \in \mathbb{R}$. Неоднородная модель Изинга изучена в работах [5], [6], [8], где дано полное описание периодических гиббсовских мер и изучены крайние точки множества предельных гиббсовских мер.

В этом пункте, используя результаты предыдуших пунктов, построим новые предельные гиббсовские меры неоднородной модели Изинга.

По построению лес Кэли является подграфом дерева Кэли.

Пусть лес Кэли $\Lambda_{k}$ состоит из деревьев Кэли $\Gamma_{i}^{k}, i=1,2, \ldots$ и $\Lambda_{k}^{(i)}-$ их множество вершин соответственно.

На $\Lambda_{k}^{(i)}$ рассмотрим гамильтониан

$$
H_{i}(\sigma)=-\sum_{\langle x, y\rangle} J_{i} \sigma(x) \sigma(y)
$$

где суммирование ведется по всем парам ближайших соседей $\langle x, y\rangle \in \Lambda_{k}^{(i)}$ и $J_{i} \in \mathbb{R}$, $i=1,2, \ldots$. На $\Lambda_{k}$ рассмотрим гамильтониан

$$
H(\sigma)=\sum_{i=1}^{\infty} H_{i}(\sigma) .
$$

Обозначим через $\omega$ число различных $J_{i}$ в (8).

Заметим, что для однородной модели Изинга $\omega=1$.

Пусть теперь для модели (8) $\omega>1$. Тогда, не нарушая общности, можно считать, что $J_{1}, J_{2}, \ldots, J_{\omega}$ различны. Обозначим $\theta_{i}=\operatorname{th}\left(J_{i} \beta\right), i=1,2, \ldots, \omega$.

ТЕОРемА 7. Если выполняются условия $\frac{1}{|I(G)|-1} \leqslant \theta_{i}<1 \quad\left(-1<\theta_{i} \leqslant\right.$ $\left.-\frac{1}{|I(G)|-1}\right), \quad i=\overline{1, \omega}$, то для каждого $\left(t_{1}, t_{2}, \ldots, t_{\omega}\right) \in D^{\omega}($ әде $D=\{(x, y) \in$ $\left.\left.[0 ; 1]^{2}: x \leqslant y\right\}\right)$ существует единственная гиббсовская мера $\mu\left(t_{1}, t_{2}, \ldots, t_{\omega}\right)$.

ДокАЗАТЕЛЬСтво. При условии теоремы существует континуум мер Гиббса на $\Gamma_{i}^{k}$, а именно для каждого $(x, y) \in D$ существует мера Гиббса $\mu(x, y)$ [10]. С помощью мер $\mu(x, y)$ анологично методам работ [6], [7], [10] можно построить $\mu\left(t_{1}, t_{2}, \ldots, t_{\omega}\right)$. Теорема доказана.

Опишем теперь новые предельные гиббсовские меры неоднородной модели Изинга на дереве Кэли, а именно докажем, что можно выбрать $J_{x, y}, x, y \in V$, так, что множество предельных гиббсовских мер будет эквивалентно множеству $D^{\omega}, \omega=$ $1,2,3, \ldots$. 
Теорема 8. Для неоднородной модели Изинга на дереве Кәли при любом $\omega=1,2, \ldots$ можно вибрать $J_{x, y}, \quad x, y \in V$, так, что для любого $\left(t_{1}, t_{2}, \ldots, t_{\omega}\right) \in D^{\omega}$ существует единственная предельная гиббсовская мера $\mu\left(t_{1}, t_{2}, \ldots, t_{\omega}\right)$.

ДокАЗАТЕЛЬСТво. Пусть $G_{k}$ - групповое представление дерева Кэли и $G$ $(|I(G)|>2)$ - некоторая подгруппа группы $G_{k}$ такая, что $G \neq G(I)$. Заметим, что $G$ представляет лес $\Lambda_{|I(G)|-1}=\left\{\Gamma_{1}^{|I(G)|-1}, \Gamma_{2}^{|I(G)|-1}, \ldots\right\}$. Пусть

$$
J_{x y}= \begin{cases}J_{i}, & \text { если }\langle x, y\rangle \in \Gamma_{i}^{|I(G)|-1}, \quad i=1,2, \ldots, \\ 0, & \text { если }\langle x, y\rangle \in G_{k} / G .\end{cases}
$$

В силу теоремы 7 для модели Изинга на дереве Кэли с параметрами (9) сушествуют меры $\mu\left(t_{1}, t_{2}, \ldots, t_{\omega}\right),\left(t_{1}, t_{2}, \ldots, t_{\omega}\right) \in D^{\omega}$. Теорема доказана.

8. Случайные блуждания в случайной среде на $F^{k}$. Пусть $F^{k}$ представляется подгруппой $G$; тогда $|I(G)|=k+1$. Зафиксируем $i$ и $j$ такие, что $a_{i}, a_{j} \in I(G)$. Рассмотрим отображения:

1) $\pi_{i j}\left(a_{m}\right)= \begin{cases}a_{m}, & \text { если } m=i, j, \\ e, & \text { если } m \neq i, j,\end{cases}$ где $e$ - единичный элемент группы $G$;

2) $f_{i j}(x)=f_{i j}\left(a_{i_{1}} a_{i_{2}} \ldots a_{i_{n}}\right)=\pi_{i j}\left(a_{i_{1}}\right) \pi_{i j}\left(a_{i_{2}}\right) \ldots \pi_{i j}\left(a_{i_{n}}\right)$, где $n=|x|$ называется длиной слова $x \in G$;

3) $g_{i j}(x)= \begin{cases}|x|, & \text { если } x=a_{i} a_{j} a_{i} a_{j} \ldots, \\ -|x|, & \text { если } x=a_{j} a_{i} a_{j} a_{i} \ldots, \\ 0, & \text { если } x=e ;\end{cases}$

4) $\phi_{i j}(x)=g_{i j}\left(f_{i j}(x)\right)$.

Легко доказать следующее

ПРЕДЛОЖЕНИЕ 3. При произвольных $i, j$

1) если $x, y \in G$ - соседние вершины, то $\phi_{i j}(x)$ и $\phi_{i j}(y)$ либо являются соседними (т.е. $\left.\phi_{i j}(x)=\phi_{i j}(y) \pm 1\right)$, либо $\phi_{i j}(x)=\phi_{i j}(y)$;

2) $\left|\phi_{i j}(x)\right| \leqslant|x|$ для любого $x \in G$.

Для любого $\varepsilon>0$ будем рассматривать совокупность векторов $\left\{p(x)=\left(p_{1}(x)\right.\right.$, $\left.\left.p_{2}(x), \ldots, p_{k+1}(x)\right), x \in G\right\}$, удовлетворяющую условиям: $\sum_{i=1}^{k+1} p_{i}(x)=1$, $p_{i}(x) \geqslant \varepsilon$ для всех $x \in G$.

Эту совокупность будем называть средой и обозначать символом $A$.

Случайным блужданием в среде $A$ называется однородная цепь Маркова $\{x(n), n=0,1,2, \ldots\}$ с матрицей переходных вероятностей $M=\{p(x, y) ; x, y \in$ $G\}$, заданной следующим образом:

$$
p(x, y)= \begin{cases}p_{i}(x), & \text { если } y=x a_{i}, \quad i=1, \ldots, k+1, \quad x \notin K(G), \\ 0.5 p_{i}(x), & \text { если } y=x a_{i} \text { или } y=a_{i} x, \quad x \in K(G), \\ 0 & \text { в остальных случаях. }\end{cases}
$$


Известно, что случайному блужданию в среде $A$ соответствует вероятностная мера $P_{A}$ на пространстве $X$ траекторий блужданий с $\sigma$-алгеброй $F$, порожденной цилиндрическими подмножествами $X$.

Рассмотрим среду как реализацию некоторого случайного процесса. Тогда $A$ зависит также от параметра $\omega$, изменяющегося на вероятностном пространстве $(\Omega, B, \mu)$.

Среда $A(\omega)=\left\{p(x)=p_{\omega}(x), x \in G\right\}$ называется случайной средой.

УСЛовиЕ П. Для любого $x \in G u y \in H_{i j}=\left\{x \in G: f_{i j}=e\right\}$ среда $A$ удовлетворяет равенству $p(x)=p(y x)$.

Заметим, что это условие есть условие периодичности среды относительно нормального делителя $H_{i j}$.

В силу предложения 3 и условия П функция $\phi_{i j}$ индуцирует случайное блуждание $\left\{x^{*}(t), t=0,1, \ldots\right\}$ с пространством состояний $Z$, начальным состоянием 0 и матрищей переходных вероятностей $M^{*}=\left\{p^{*}(x, y) ; x, y \in Z\right\}$, заданной следующим образом:

$$
p^{*}(x, y)= \begin{cases}p_{i}^{*}(x), & \text { если } y=x+1, \\ p_{j}^{*}(x), & \text { если } y=x-1, \\ q_{i j}^{*}(x), & \text { если } y=x, \\ 0 & \text { в остальных случаях, }\end{cases}
$$

где $q_{i j}^{*}(x)=1-p_{i}^{*}(x)-p_{j}^{*}(x)$ и $p_{m}^{*}(x)=\sum_{l: \phi_{i j}\left(x a_{l}\right)=\phi_{i j}\left(x a_{m}\right)} p_{l}(x), m=i, j$.

Обозначим $D_{i j}=\mathrm{E}_{\mu} \ln \frac{p_{j}^{*}(x)}{p_{i}^{*}(x)}$, где $\mathrm{E}_{\mu}$ - математическое ожидание по мере $\mu$.

ТЕОРема 9. Если существуют такие $i, j$, для которых выполняется условие $\Pi$, mo

1) при $D_{i j} \neq 0$

$$
P_{A}\left\{\lim _{n \rightarrow \infty}|x(n)|=+\infty\right\}=1 \quad(\mu-n . \boldsymbol{\theta} .)
$$

2) при $D_{i j}=0$

$$
P_{A}\left\{\limsup _{n \rightarrow \infty}|x(n)|=+\infty\right\}=1 \quad(\mu-n . \text { в. })
$$

ДоКАЗАТЕЛЬСТво. Из определения $\phi_{i j}$ в силу предложения 3 ясно, что для любого $n=0,1, \ldots$

$$
\left|x^{*}(n)\right| \leqslant|x(n)| \text {. }
$$

Но для $x^{*}(n)$ теорема верна (см. [12], [13]), следовательно, в силу (10) получаем утверждения 1) и 2). Теорема доказана. 


\section{Список литературы}

1. Ганиходжаев H. Н. Групповое представление и автоморфизмы дерева Кэли // ДАН РУ 3. 1994. № 4. С. 3-5.

2. Пирогов С.А., Синай Я. Г. Фазовые диаграммы классических решетчатых систем. I // ТМФ. 1975. Т. 25. №3. C. 369-378.

3. Ганиходжаев Н.Н., Розиков У.А. Описание периодических крайных гиббсовских мер некоторых моделей на дереве Кэли // ТМФ. 1997. Т. 111. № 1. С. 109-117.

4. Пирогов С. А., Синай Я.Г. Фазовые диаграммы классических решетчатых систем. II // ТМФ. 1976. Т. 26. №1. С. 61-76.

5. Розиков У.А. Структура разбиений группового представления дерева Кэли и их применения для описания периодических распределений Гиббса // ТМФ. 1997. Т. 112. № 1. C. $170-176$.

6. Розиков У.А. Построение несчетного числа гиббсовских мер неоднородной модели Изинга // ТМФ. 1999. Т. 118. № 1. С. 95-104.

7. Розиков У.А. Описание предельных гиббсовских мер $\lambda$-моделей на решетке Бете // CMЖ. 1998. Т. 39. № 2. C. 427-435.

8. Ганиходжаев H.Н., Розиков У.А. О неупорядоченных фазах некоторых моделей на дереве Кэли // Матем. сб. 1999. Т. 190. № 2. С. 31-42.

9. Ganikhodjaev N. N., Rozikov U. A. On disordered phase of ferromagnetic Potts model on the Bethe lattece // Osaka J. of Math. 2000. V. 37. № 2. P. 373-383.

10. Блехер П. М., Ганиходжсаев Н. Н. О чистых фазах модели Изинга на дереве Кэли // ТВП. 1990. Т. 35. № 2. С. 920-930.

11. Синай Я.Г. Теория фазовых переходов. М.: Наука, 1980.

12. Летчиков $A . B$. Критерий применимости центральной предельной теоремы к однородным случайным блужданиям в случайных средах // ТВП. 1992. Т. 32. № 3. С. 576-580.

13. Solomon F. Random walks in random environment // Ann. Prob. 1975. V. 3. № 1. P. 1-31.

E-mail: root@im.tashkent.su

Поступило в редакцию

27.VI. 2001 